Proyecciones Journal of Mathematics

Vol. 37, No 4, pp. 613-625, December 2018.

Universidad Católica del Norte

Antofagasta - Chile

\title{
Odd Vertex equitable even labeling of cyclic snake related graphs
}

\author{
P. Jeyanthi \\ Govindammal Aditanar College for Women, India \\ and \\ A. Maheswari \\ Kamaraj College of Engineering and Technology, India \\ Received: January 2018. Accepted: March 2018
}

\begin{abstract}
Let $G$ be a graph with $p$ vertices and $q$ edges and $A=\{1,3, \ldots, q\}$ if $q$ is odd or $A=\{1,3, \ldots, q+1\}$ if $q$ is even. A graph $G$ is said to admit an odd vertex equitable even labeling if there exists a vertex labeling $f: V(G) \rightarrow A$ that induces an edge labeling $f^{*}$ defined by $f^{*}(u v)=f(u)+f(v)$ for all edges uv such that for all $a$ and $b$ in $A$, $\left|v_{f}(a)-v_{f}(b)\right| \leq 1$ and the induced edge labels are $2,4, \ldots, 2 q$ where $v_{f}(a)$ be the number of vertices $v$ with $f(v)=a$ for $a \in A$. A graph that admits an odd vertex equitable even labeling is called an odd vertex equitable even graph. Here, we prove that the graph $n C_{4}$-snake, $C S\left(n_{1}, n_{2}, \ldots, n_{k}\right), n_{i} \equiv 0(\bmod 4), n_{i} \geq 4$, be a generalized $k C_{n}$-snake, $T \widehat{O} Q S_{n}$ and $T \widetilde{O} Q S_{n}$ are odd vertex equitable even graphs.
\end{abstract}

Keywords : vertex equitable labeling, vertex equitable graph, odd vertex equitable even labeling, odd vertex equitable even graph.

AMS Subject Classification (2010) : 05C78 


\section{Introduction}

All graphs considered here are simple, finite, connected and undirected. Let $G(V, E)$ be a graph with $p$ vertices and $q$ edges. We follow the basic notations and terminology of graph theory as in [3]. A graph labeling is an assignment of integers to the vertices or edges or both, subject to certain conditions and a detailed survery of graph labeling can be found in [2]. The vertex set and the edge set of a graph are denoted by $V(G)$ and $E(G)$ respectively.

The concept of vertex equitable labeling was due to Lourdusamy and Seenivasan in [16] and further studied in [5]-[14]. Let $G$ be a graph with $p$ vertices and $q$ edges and $A=\left\{0,1,2, \ldots,\left\lceil\frac{q}{2}\right\rceil\right\}$. A graph $G$ is said to be vertex equitable if there exists a vertex labeling $f: V(G) \rightarrow A$ that induces an edge labeling $f^{*}$ defined by $f^{*}(u v)=f(u)+f(v)$ for all edges $u v$ such that for all $a$ and $b$ in $A,\left|v_{f}(a)-v_{f}(b)\right| \leq 1$ and the induced edge labels are $1,2,3, \ldots, q$, where $v_{f}(a)$ is the number of vertices $v$ with $f(v)=a$ for $a \in A$. The vertex labeling $f$ is known as vertex equitable labeling. A graph $G$ is said to be a vertex equitable if it admits a vertex equitable labeling.

Motivated by the concept of vertex equitable labeling of graphs, Jeyanthi, Maheswari and Vijaya Lakshmi defined a new labeling namely odd vertex equitable even labeling [15]. A graph $G$ with $p$ vertices and $q$ edges and $A=\{1,3, \ldots, q\}$ if $q$ is odd or $A=\{1,3, \ldots, q+1\}$ if $q$ is even. A graph $G$ is said to admit an odd vertex equitable even labeling if there exists a vertex labeling $f: V(G) \rightarrow A$ that induces an edge labeling $f^{*}$ defined by $f^{*}(u v)=f(u)+f(v)$ for all edges $u v$ such that for all $a$ and $b$ in $A$, $\left|v_{f}(a)-v_{f}(b)\right| \leq 1$ and the induced edge labels are $2,4, \ldots, 2 q$ where $v_{f}(a)$ be the number of vertices $v$ with $f(v)=a$ for $a \in A$. A graph that admits an odd vertex equitable even labeling is called an odd vertex equitable even graph. In [15] they proved that the graphs like path, $P_{n} \odot P_{m}(n, m \geq 1)$, $K_{1, n} \cup K_{1, n-2}(n \geq 3), K_{2, n}, T_{p}$-tree, a ladder $L_{n}$, arbitrary super subdivision of any path $P_{n}$ are odd vertex equitable even graphs.

Also they proved that the graphs $K_{1, n}$ is an odd vertex equitable even graph iff $n \leq 2$, the graph $G=K_{1, n} \cup K_{1, n-2}(n \geq 3)$ is an odd vertex equitable even graph and cycle $C_{n}$ is an odd vertex equitable even graph if $n \equiv 0$ or $1(\bmod 4)$. In addition, they proved that if every edge of a graph $G$ is an edge of a triangle, then $G$ is not an odd vertex equitable even graph. 
We use the following definitions in the subsequent section.

Theorem 1.1. The cycle $C_{n}$ is an odd vertex equitable even graph if $n \equiv 0$ or $1(\bmod 4)$.

Theorem 1.2. Let $G_{1}\left(p_{1}, q_{1}\right), G_{2}\left(P_{2}, q_{2}\right), \ldots, G_{m}\left(p_{m}, q_{m}\right)$ be an odd vertex equitable even graphs with $\sum_{i=1}^{m-1} q_{i}$ is even, $q_{m}$ is even or odd and $u_{i}, v_{i}$ be the vertices of $G_{i}(1 \leq i \leq m)$ labeled by $1, q_{i}$ if $q_{i}$ is odd or $q_{i}+1$ if $q_{i}$ is even. Then the graph $G$ obtained by identifying $v_{1}$ with $u_{2}$ and $v_{2}$ with $u_{3}$ and $v_{3}$ with $u_{4}$ and so on until we identify $v_{m-1}$ with $u_{m}$ is also an odd vertex equitable even graph.

Definition 1.3. The corona $G_{1} \odot G_{2}$ of the graphs $G_{1}$ and $G_{2}$ is defined as a graph obtained by taking one copy of $G_{1}$ (with $p$ vertices) and $p$ copies of $G_{2}$ and then joining the $i^{\text {th }}$ vertex of $G_{1}$ to every vertex of the $i^{\text {th }}$ copy of $G_{2}$.

Definition 1.4. Let $G_{1}$ be a graph with $p$ vertices and $G_{2}$ be any graph. A graph $G_{1} \widehat{o} G_{2}$ is obtained from $G_{1}$ and $p$ copies of $G_{2}$ by identifying one vertex of $i^{\text {th }}$ copy of $G_{2}$ with $i^{\text {th }}$ vertex of $G_{1}$.

Definition 1.5. [1] $A k C_{n}$-snake is defined as a connected graph in which all the $k$-blocks are isomorphic to the cycle $C_{n}$ and the block-cut point graph is a path. Let $P$ be the path of minimum length that contains all the cut vertices of a $k C_{n}$-snake. Barrientos proved that any $k C_{n}$-snake is represented by a string $s_{1}, s_{2}, \ldots, s_{k-2}$ of integers of length $k-2$ where the $i^{\text {th }}$ integer, $s_{i}$ on the string is the distance between $i^{\text {th }}$ and $(i+1)^{\text {th }}$ cut vertices on the path $P$ from one extreme and is taken from $S_{n}=\left\{1,2, \ldots,\left\lfloor\frac{n}{2}\right\rfloor\right\}$. The strings obtained for both extremes are assumed to be the same. Then there are at most $\left\lfloor\frac{n}{2}\right\rfloor^{k-2}$ non isomorphic $k C_{n}$-snakes. For example, the string of a $10 C_{4}$-snake is shown in Figure 1.1 is 2,2,1,2,1,1,2,1. $A k C_{n}$-snake is said to be linear if each integer of its string is $\left\lfloor\frac{n}{2}\right\rfloor$. 


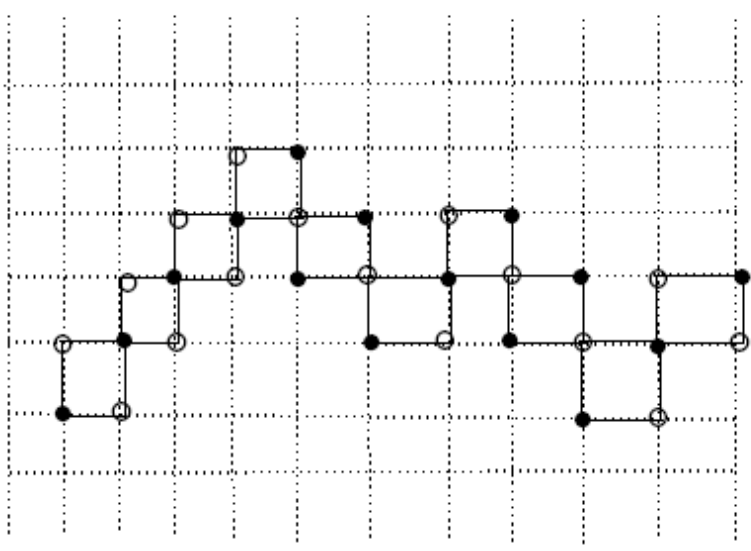

Figure 1.1: An embedding of $10 C_{4}$-snake

A $n C_{k}$-snake is said to be linear if each integer of its string is $\left\lfloor\frac{k}{2}\right\rfloor$. The linear $n C_{4}$-snake graph with diagonal vertices $u_{1 j}(1 \leq j \leq n+1)$, left to the diagonal vertices $v_{1 j}(1 \leq j \leq n)$ and right to the diagonal vertices $w_{1 j}$ $(1 \leq j \leq n)$ is denoted by $Q S_{n}$. For example, a linear $3 C_{4}$-snake graph $Q S_{3}$ is shown in Figure 1.2.

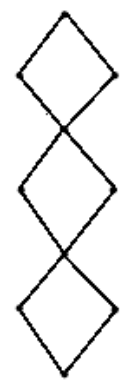

Figure 1.2: A linear $3 C_{4}$-snake $Q S_{3}$

Definition 1.6. A generalized $k C_{n}$-snake is defined as a connected graph in which each block is isomorphic to a cycle $C_{n}$ for some $n$ and the block-cut point graph is a path. It is denoted by $C S\left(n_{1}, n_{2}, \ldots, n_{k}\right)$ where $B_{1}, B_{2}, \ldots, B_{k}$ are the consecutive blocks and $B_{i}$ is isomorphic to $C_{n_{i}}$. By applying the same methods used to obtain the strings of a $k C_{n}$-snake, we can show that any generalized $k C_{n}$-snake can also be represented by a string of integers $s_{1}, s_{2}, \ldots, s_{k-2}$ of length $k-2$ where $s_{i-1} \in S_{n_{i}}$. 
Definition 1.7. [4] Let $T$ be a tree and $u_{0}$ and $v_{0}$ be the two adjacent vertices in $T$. Let $u$ and $v$ be the two pendant vertices of $T$ such that the length of the path $u_{0}-u$ is equal to the length of the path $v_{0}-v$. If the edge $u_{0} v_{0}$ is deleted from $T$ and $u$ and $v$ are joined by an edge $u v$, then such a transformation of $T$ is called an elementary parallel transormation (or an ept) and the edge $u_{0} v_{0}$ is called transformable edge. If by the sequence of ept's, $T$ can be reduced to a path, then $T$ is called a $T_{p}$-tree (transformed tree) and such sequence regarded as a composition of mappings (ept's) denoted by $P$, is called a parallel transformation of $T$. The path, the image of $T$ under $P$ is denoted as $P(T)$. A $T_{p}$-tree and the sequence of two ept's reducing it to a path are illustrated in Figure 1.3.
(a) $A T_{p}$-tree $T$
(b) An ept $P_{1}(T)$
(c) Second ept $P_{2}(T)$
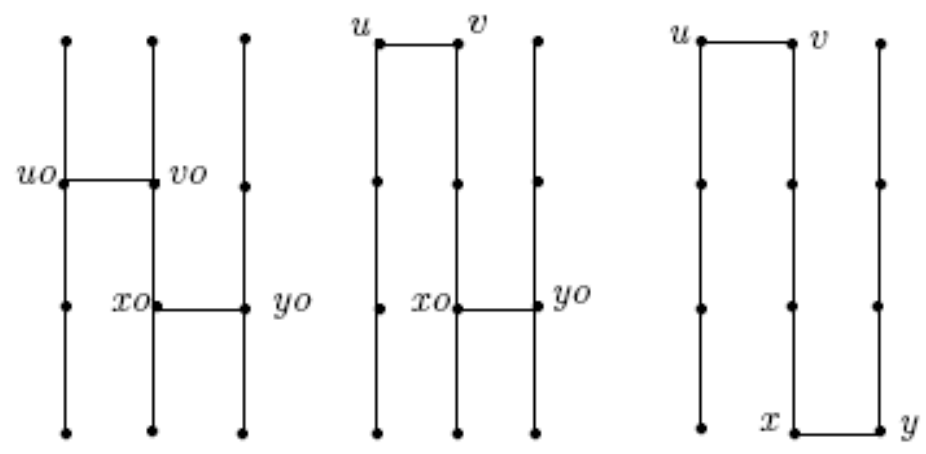

Figure 1.3

\section{Main Results}

In this section, we prove that $n C_{4}$-snake, $C S\left(n_{1}, n_{2}, \ldots, n_{k}\right), n_{i} \equiv 0(\bmod 4)$, $n_{i} \geq 4$, be a generalized $k C_{n}$-snake, $T \widehat{O} Q S_{n}$ and $T \widetilde{O} Q S_{n}$ are odd vertex equitable even graphs.

Theorem 2.1. The $\mathrm{nC}_{4}$-snake is an odd vertex equitable even graph.

Proof. Let $G$ be a $n C_{4}$-snake with $n$ blocks and $G_{i}=C_{4}, 1 \leq i \leq n-1$ and $u_{i}, v_{i}$ be the vertices with labels 1 and $q+1$ respectively. By Theorem 1.2, $n C_{4}$ admits an odd vertex equitable even labeling. An example for odd vertex equitable even labeling of $3 C_{4}$-snake is shown in Figure 2.1. 


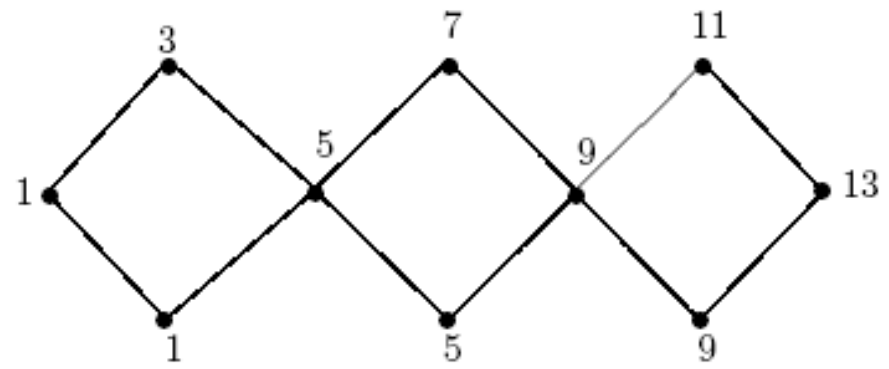

Figure 2.1

Theorem 2.2. Let $G=C S\left(n_{1}, n_{2}, \ldots, n_{k}\right), n_{i} \equiv 0(\bmod 4), n_{i} \geq 4$ be a generalized $k C_{n}$-snake with its strings $s_{1}, s_{2}, \ldots, s_{k-2}$ where $s_{i} \in\{1\}, 1 \leq$ $i \leq k$. Then $G$ is an odd vertex equitable even graph.

Proof. By Theorem 1.1, the cycle $C_{n}$ is an odd vertex equitable even graph if $n \equiv 0(\bmod 4)$. By Theorem 1.2, $C S\left(n_{1}, n_{2}, \ldots, n_{k}\right), n_{i} \equiv 0(\bmod 4)$, is an odd vertex equitable even graph. An example for odd vertex equitable even labeling of $C S(8,4,12)$ is shown in Figure 2.2.

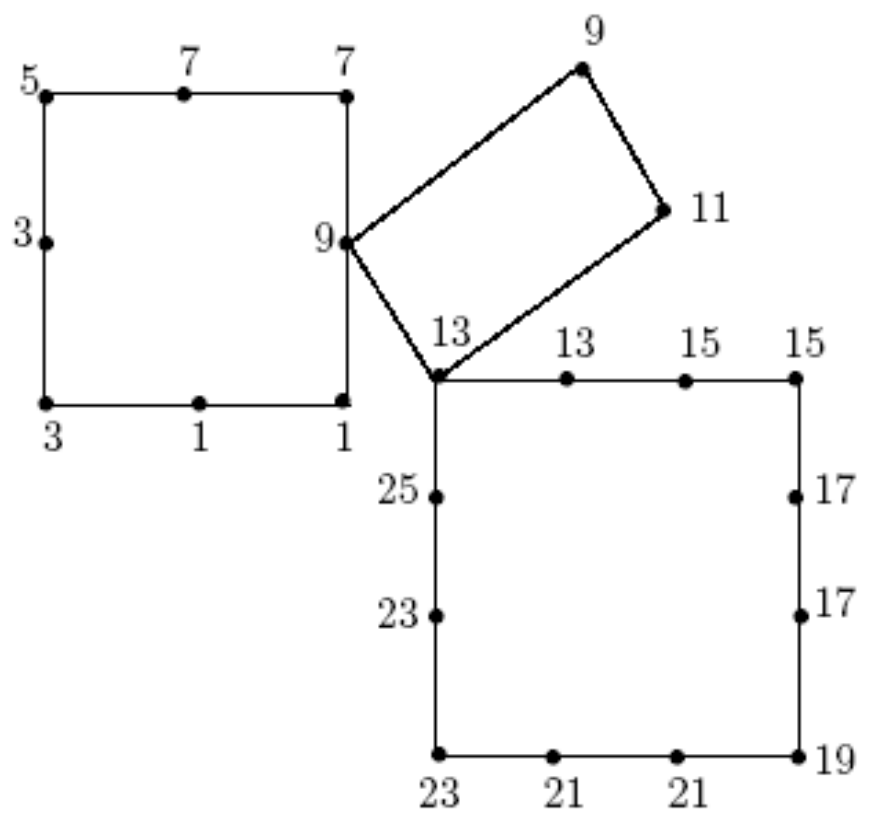

Figure 2.2 
Theorem 2.3. If $T$ be a $T_{p}$-tree on $m$ vertices, then the graph $T \widehat{O} Q S_{n}$ is an odd vertex equitable even graph.

Proof. $\quad$ Let $T$ be a $T_{p}$-tree with $m$ vertices. By the definition of a transformed tree there exists a parallel transformation $P$ of $T$ such that for the path $P(T)$ we have (i) $V(P(T))=V(T)$ (ii) $E(P(T))=\left(E(T)-E_{d}\right) \cup E_{p}$ where $E_{d}$ is the set of edges deleted from $T$ and $E_{p}$ is the set of edges newly added through the sequence $P=\left(P_{1}, P_{2}, \ldots, P_{k}\right)$ of the epts $P$ used to arrive at the path $P(T)$. Clearly, $E_{d}$ and $E_{p}$ have the same number of edges.

Now denote the vertices of $P(T)$ successively by $u_{1}^{\prime}, u_{2}^{\prime}, \ldots, u_{m}^{\prime}$ starting from one pendant vertex of $P(T)$ right up to the other one.

Let $u_{i 1}, u_{i 2}, \ldots, u_{i(n+1)}, v_{i 1}, v_{i 2}, \ldots, v_{i n}$ and $w_{i 1}, w_{i 2}, \ldots, w_{i n}(1 \leq i \leq m)$ be the vertices of $i^{\text {th }}$ copy of $P_{n}$ with $u_{i(n+1)}=u_{i}^{\prime}$.

Then $V\left(T \widehat{O} Q S_{n}\right)=\left\{u_{i j}: 1 \leq i \leq m, 1 \leq j \leq n+1\right.$ with $u_{i(n+1)}=$ $\left.u_{i}^{\prime}\right\} \bigcup\left\{u_{i}^{\prime}, v_{i j}, w_{i j}: 1 \leq i \leq m, 1 \leq j \leq n\right\}$ and $E\left(T \widehat{O} Q S_{n}\right)=\left\{e_{i}^{\prime}=u_{i}^{\prime} u_{i+1}^{\prime}: 1 \leq i \leq m-1\right\} \cup E\left(Q S_{n}\right)$.

Here $\left|V\left(T \widehat{O} Q S_{n}\right)\right|=m(3 n+1)$ and $\left|E\left(T \widehat{O} Q S_{n}\right)\right|=4 m n+m-1$.

Let $A=\{1,3, \ldots, 4 m n+m-1\}$.

Define a vertex labeling $f: V\left(T \widehat{O} Q S_{n}\right) \rightarrow A$ as follows:

For $1 \leq i \leq m, 1 \leq j \leq n+1 f\left(u_{i j}\right)=\begin{array}{ll}(4 n+1)(i-1)+4 j-3 & \text { if } i \text { is odd } \\ (4 n+1) i-(4 j-3) & \text { if } i \text { is even }\end{array}$.

For $1 \leq i \leq m, 1 \leq j \leq n$.

$$
f\left(v_{i j}\right)=f\left(u_{i j}\right), f\left(w_{i j}\right)=\begin{array}{ll}
(4 n+1)(i-1)+4 j-1 & \text { if } i \text { is odd } \\
(4 n+1) i-(4 j-1) & \text { if } i \text { is even }
\end{array} .
$$

For the vertex labeling $f$, the induced edge labeling $f^{*}$ is as follows:

For $1 \leq i \leq m-1 f^{*}\left(e_{i}^{\prime}\right)=2(4 n+1) i$.

The induced edge labels of $Q S_{n}$ are $2(4 n+1)(i-1)+2 j(1 \leq i \leq m, 1 \leq$ $j \leq 2 n)$ if $i$ is odd and $2(4 n+1)(i-1)+2 j(1 \leq i \leq m, 1 \leq j \leq 2 n)$ if $i$ is even.

Let $v_{i} v_{j}$ be a transformed edge in $T$ for some indices $i, j, 1 \leq i \leq j \leq m$. 
Let $P_{1}$ be the ept that deletes the edge $v_{i} v_{j}$ and adds an edge $v_{i+t} v_{j-t}$ where $t$ is the distance of $v_{i}$ from $v_{i+t}$ and the distance of $v_{j}$ from $v_{j-t}$.

Let $P$ be a parallel transformation of $T$ that contains $P_{1}$ as one of the constituent epts. Since $v_{i+t} v_{j-t}$ is an edge in the path $P(T)$, it follows that $i+t+1=j-t$ which implies $j=i+2 t+1$.

Therefore, $i$ and $j$ are of opposite parity, that is, $i$ is odd and $j$ is even or vice-versa.

The induced label of the edge $v_{i} v_{j}$ is given by $f^{*}\left(v_{i} v_{j}\right)=f^{*}\left(v_{i} v_{i+2 t+1}\right)=$ $f\left(v_{i}\right)+f\left(v_{i+2 t+1}\right)=2(4 n+1)(i+t)$ and

$$
f^{*}\left(v_{i+t} v_{j-t}\right)=f^{*}\left(v_{i+t} v_{i+t+1}\right)=f\left(v_{i+t}\right)+f\left(v_{i} v_{i+t+1}\right)=2(4 n+1)(i+t) .
$$

Therefore, $f^{*}\left(v_{i} v_{j}\right)=f^{*}\left(v_{i+t} v_{j-t}\right)$. It can be verified that the induced edge labels of $T \widehat{O} Q S_{n}$ are $2,4,6, \ldots, 8 m n+2 m-2$ and $\left|v_{f}(a)-v_{f}(b)\right| \leq 1$ for all $a, b \in A$.

Hence, $T \widehat{O} Q S_{n}$ is an odd vertex equitable even graph.

An example for odd vertex equitable even labeling of $T \widehat{O} Q S_{2}$ where $T$ is a $T_{p}$-tree on 8 vertices is shown in Figure 2.3. 


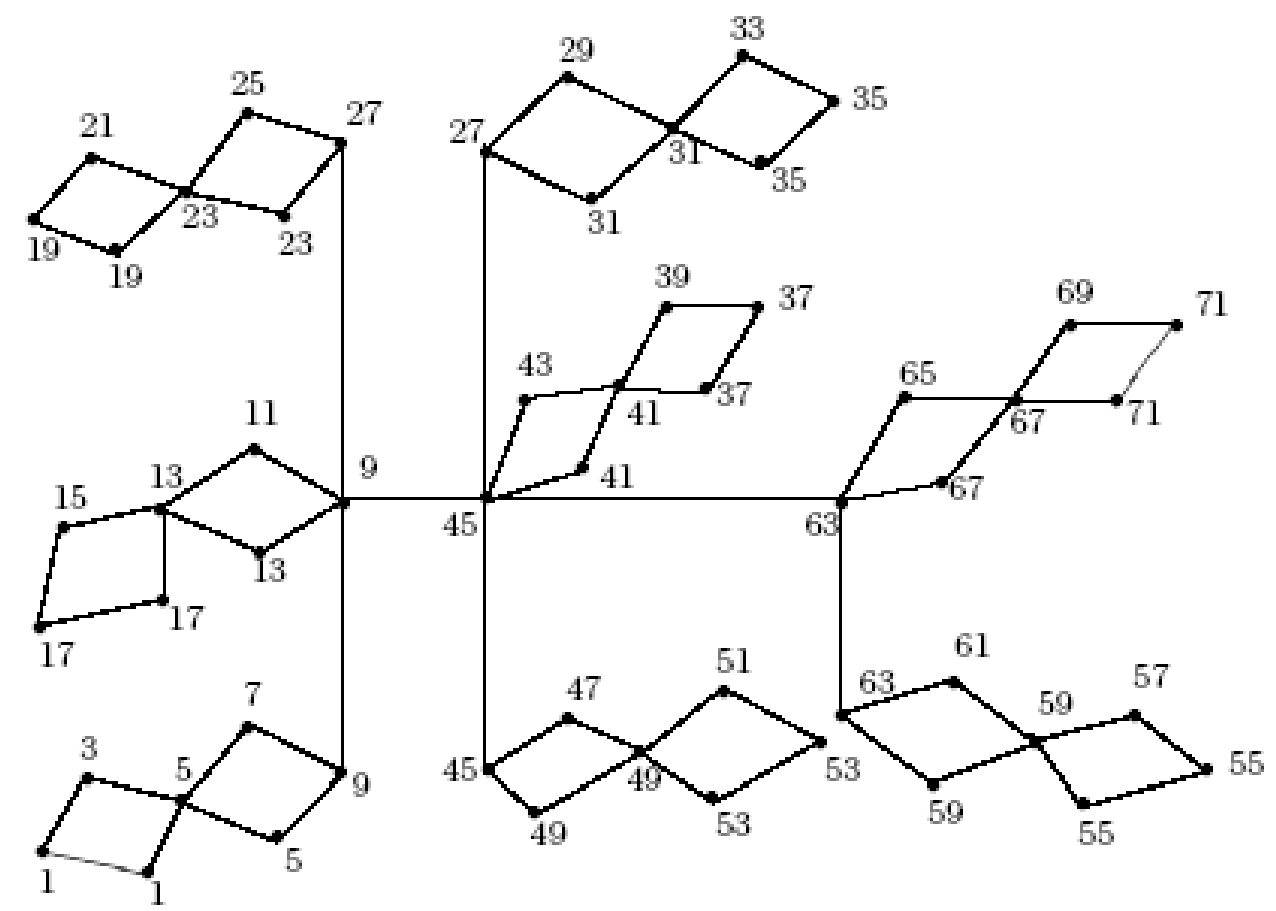

Figure 2.3

Theorem 2.4. Let $T$ be a $T_{p}$-trees on $m$ vertices. Then the graph $T \widetilde{O} Q S_{n}$ is an odd vertex equitable even graph.

Proof. $\quad$ Let $T$ be a $T_{p}$-tree with $m$ vertices. By the definition of a transformed tree there exists a parallel transformation $P$ of $T$ such that for the path $P(T)$ we have (i) $V(P(T))=V(T)$ (ii) $E(P(T))=\left(E(T)-E_{d}\right) \cup E_{p}$ where $E_{d}$ is the set of edges deleted from $T$ and $E_{p}$ is the set of edges newly added through the sequence $P=\left(P_{1}, P_{2}, \ldots, P_{k}\right)$ of the epts $P$ used to arrive at the path $P(T)$. Clearly, $E_{d}$ and $E_{p}$ have the same number of edges. Now denote the vertices of $P(T)$ successively by $u_{1}^{\prime}, u_{2}^{\prime}, \ldots, u_{m}^{\prime}$ starting from one pendant vertex of $P(T)$ right up to the other one.

Let $u_{i 1}, u_{i 2}, \ldots, u_{i(n+1)}, v_{i 1}, v_{i 2}, \ldots, v_{i n}$ and $w_{i 1}, w_{i 2}, \ldots, w_{i n}(1 \leq i \leq m)$ be the vertices of $i^{\text {th }}$ copy of $P_{n}$. 
Then $V\left(T \widetilde{O} Q S_{n}\right)=\left\{u_{i j}: 1 \leq i \leq m, 1 \leq j \leq n+1\right\} \bigcup\left\{u_{i}^{\prime}, v_{i j}, w_{i j}\right.$ : $1 \leq i \leq m, 1 \leq j \leq n\}$ and $E\left(T \widetilde{O} Q S_{n}\right)=E\left(Q S_{n}\right) \bigcup\left\{e_{i}^{\prime}=u_{i}^{\prime} u_{i+1}^{\prime}: 1 \leq i \leq\right.$ $m-1\} \bigcup\left\{e_{i}^{\prime \prime}=u_{i}^{\prime} u_{i(n+1)}: 1 \leq i \leq m\right\}$.

Here $\left|V\left(T \widetilde{O} Q S_{n}\right)\right|=m(3 n+2)$ and $\left|E\left(T \widetilde{O} Q S_{n}\right)\right|=4 m n+2 m-1$.

Let $A=\{1,3, \ldots, 4 m n+2 m-1\}$.

Define a vertex labeling $f: V\left(T \widetilde{O} Q S_{n}\right) \rightarrow A$ as follows:

For $1 \leq i \leq m, 1 \leq j \leq n+1 f\left(u_{i j}\right)=\begin{array}{ll}(4 n+2)(i-1)+4 j-3 & \text { if } i \text { is odd } \\ (4 n+2) i-(4 j-3) & \text { if } i \text { is even }\end{array}$.

For $1 \leq i \leq m, 1 \leq j \leq n$.

$f\left(v_{i j}\right)=f\left(u_{i j}\right), f\left(w_{i j}\right)=\begin{array}{ll}(4 n+2)(i-1)+4 j-1 & \text { if } i \text { is odd } \\ (4 n+2) i-(4 j-1) & \text { if } i \text { is even } .\end{array}$

$f\left(u_{i}^{\prime}\right)=\begin{array}{ll}(4 n+2) i-1 & \text { if } i \text { is odd } \\ (4 n+2) i-(4 n+1) & \text { if } i \text { is even }\end{array}$.

For the vertex labeling $f$, the induced edge labeling $f^{*}$ is as follows:

For $1 \leq i \leq m-1 f^{*}\left(e_{i}^{\prime}\right)=2(4 n+2) i$,

For $1 \leq i \leq m f^{*}\left(e_{i}^{\prime \prime}\right)=\begin{array}{ll}2(4 n+2) i-2 & \text { if } i \text { is odd } \\ 2(4 n+2)(i-1)+2 & \text { if } i \text { is even }\end{array}$.

The induced edge labels of $Q S_{n}$ are $2(4 n+2)(i-1)+2 j(1 \leq i \leq m, 1 \leq$ $j \leq 2 n)$ if $i$ is odd and $2(4 n+2)(i-1)+2 j(1 \leq i \leq m, 1 \leq j \leq 2 n)$ if $i$ is even.

Let $v_{i} v_{j}$ be a transformed edge in $T$ for some indices $i, j, 1 \leq i \leq j \leq m$.

Let $P_{1}$ be the ept that deletes the edge $v_{i} v_{j}$ and adds an edge $v_{i+t} v_{j-t}$ where $t$ is the distance of $v_{i}$ from $v_{i+t}$ and the distance of $v_{j}$ from $v_{j-t}$.

Let $P$ be a parallel transformation of $T$ that contains $P_{1}$ as one of the constituent epts. Since $v_{i+t} v_{j-t}$ is an edge in the path $P(T)$, it follows that $i+t+1=j-t$ which implies $j=i+2 t+1$.

Therefore, $i$ and $j$ are of opposite parity, that is, $i$ is odd and $j$ is even or vice-versa.

The induced label of the edge $v_{i} v_{j}$ is given by $f^{*}\left(v_{i} v_{j}\right)=f^{*}\left(v_{i} v_{i+2 t+1}\right)=$ $f\left(v_{i}\right)+f\left(v_{i+2 t+1}\right)=2(4 n+2)(i+t)$ and 


$$
f^{*}\left(v_{i+t} v_{j-t}\right)=f^{*}\left(v_{i+t} v_{i+t+1}\right)=f\left(v_{i+t}\right)+f\left(v_{i} v_{i+t+1}\right)=2(4 n+2)(i+t) .
$$

Therefore, $f^{*}\left(v_{i} v_{j}\right)=f^{*}\left(v_{i+t} v_{j-t}\right)$.

It can be verified that the induced edge labels of $T \widetilde{O} Q S_{n}$ are $2,4,6, \ldots, 8 m n+$ $4 m-2$ and $\left|v_{f}(a)-v_{f}(b)\right| \leq 1$ for all $a, b \in A$.

Hence, $T \widetilde{O} Q S_{n}$ is an odd vertex equitable even graph.

An example for odd vertex equitable even labeling of $T \widetilde{O} Q S_{2}$ where $T$ is a $T_{p}$-tree on 8 vertices is shown in Figure 2.4.

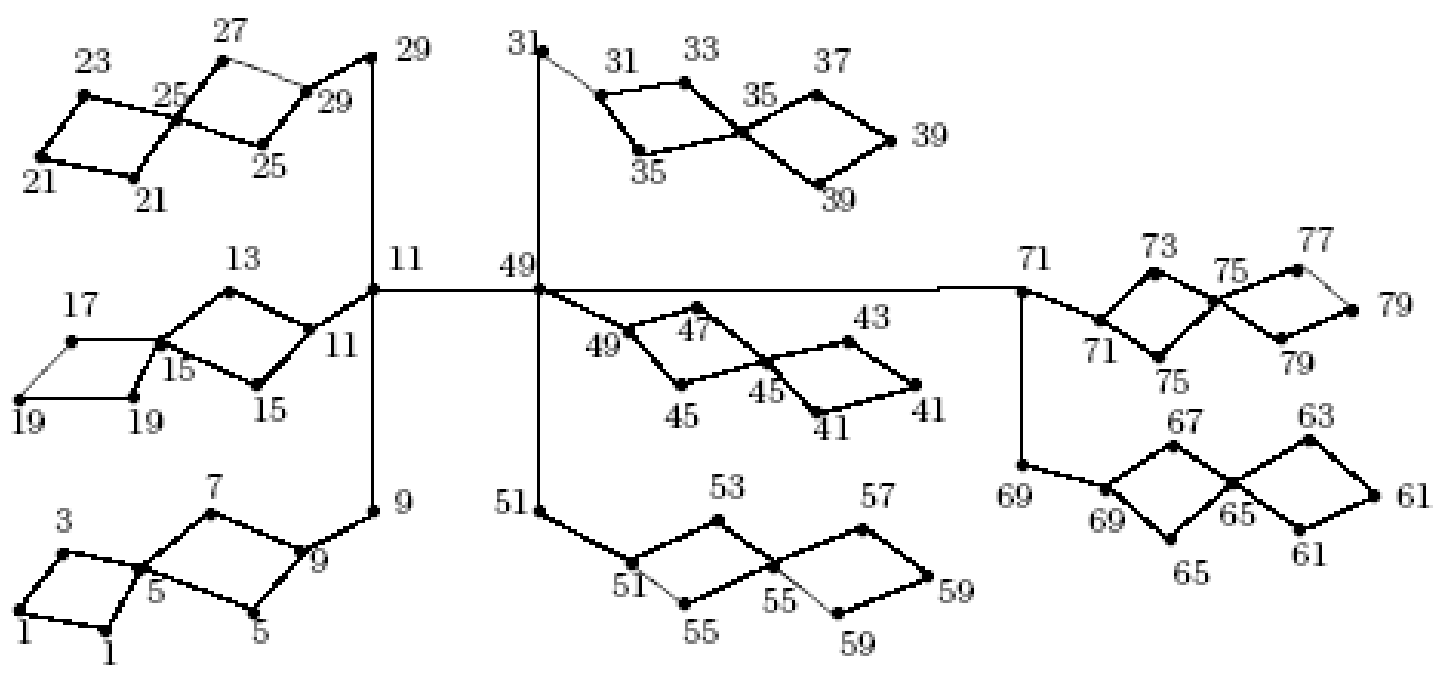

Figure 2.4 


\section{References}

[1] C. Barrientos, Difference Vertex Labelings, Ph.D. Thesis, Universitat Politecnica de Catalunya, Barcelona, (2004).

[2] Joseph A. Gallian, A Dynamic Survey of Graph Labeling, The Electronic Journal of Combinatorics, 19, (2017)\#DS6.

[3] F. Harary, Graph theory, Addison Wesley, Massachusetts, (1972).

[4] S. M. Hedge and Sudhakar Shetty, On Graceful Trees, Applied Mathematics E-Notes, 2, pp. 192-197, (2002).

[5] P. Jeyanthi and A. Maheswari, Some results on vertex equitable labeling, Open J. Discrete Math., 2, pp. 51-57, (2012).

[6] P. Jeyanthi and A. Maheswari, Vertex equitable labeling of Transformed Trees, Journal of Algorithms and Computation, 44, pp. 9-20, (2013).

[7] P. Jeyanthi and A. Maheswari, Vertex equitable labeling of cyclic snakes and bistar graphs, Journal of Scientific Research, 6, (1), pp. 79-85, (2014).

[8] P. Jeyanthi and A. Maheswari, Vertex equitable labeling of families of graphs, Graph theory Notes of New York, LXVII, pp. 34-42, (2014).

[9] P.Jeyanthi and A. Maheswari, Vertex Equitable Labeling of Super Subdivision Graphs, Scientific International, 27 (4), pp. 1-3, (2015).

[10] P. Jeyanthi, A. Maheswari and M. Vijayalakshmi, Vertex equitable labeling of cycle and star related graphs, Journal of Scientifc Research, 7, pp. 33-42, (2015).

[11] P. Jeyanthi and A. Maheswari, Vertex equitable labeling of cycle and path related graphs, Utilitas Mathematica, 98, pp. 215-226, (2015).

[12] P. Jeyanthi, A. Maheswari and M. Vijaya Lakshmi, Vertex equitable labeling of Double Alternate Snake Graphs, Journal of Algorithms and Computation, 46, pp. 27-34, (2015). 
[13] P. Jeyanthi, A. Maheswari and M. Vijaya Lakshmi, New Results on Vertex Equitable Labeling, Journal of Algebra Combinatorics Discrete structures and Applications, 3, (2), pp. 97-104, (2016).

[14] P.Jeyanthi, A. Maheswari and M. Vijaya Lakshmi, Vertex Equitable Labeling of Union of Cyclic Snake graphs, Proyecciones Journal of Mathematics, 35, (2), pp. 177-186, (2016).

[15] P. Jeyanthi, A. Maheswari and M. Vijaya Lakshmi, Odd Vertex Equitable Even Labeling, Proyecciones Journal of Mathematics, 36, (1), pp. 1-11, (2017).

[16] A. Lourdusamy and M. Seenivasan, Vertex equitable labeling of graphs, Journal of Discrete Mathematical Sciences and Cryptography, 11, (6), pp. 727-735, (2008).

\title{
P. Jeyanthi
}

Research Centre,

Department of Mathematics,

Govindammal Aditanar College for Women,

Tiruchendur-628215, Tamilnadu,

India

e-mail : jeyajeyanthi@rediffmail.com

and

\author{
A. Maheswari \\ Department of Mathematics, \\ Kamaraj College of Engineering and Technology, \\ Virudhunagar, Tamilnadu, \\ India \\ e-mail : bala_nithin@yahoo.co.in
}

